\section{Baitolo, una doble inscripción ibérica en un cepo de ancla de plomo del siglo I a.C.}

Baitolo, a double Iberian
inscription on a lead anchor
stock from the $1^{\text {st }} c . B C$

\author{
Joan Ferrer i Jané \\ Universitat de Barcelona \\ joan.ferrer.i.jane@gmail.com
}

Alejandro G. Sinner
University of Victoria
agsinner@uvic.ca

Resumen: El cepo de ancla de la colección Guerra es el primero de su categoría que presenta una inscripción ibérica: baitolo, probablemente un topónimo, bien el nombre de la ciudad de baitolo/Baetulo, la actual Badalona, que emite monedas con la leyenda baitolo en el $2^{\circ}$ cuarto del s. I a.C., o bien el del río próximo, el actual Besós. Las inscripciones griegas y romanas sobre cepos contienen básicamente nombres de armadores y de divinidades, que coinciden en este último caso con el nombre de la nave al que el cepo perteneció. Esta última práctica permitiría esperar también un topónimo, puesto que los nombres de naves de origen geográfico son frecuentes. La nave que llevaba esta ancla se construyó a mediados del s. I a.C., y probablemente perteneció a un armador layetano de la ciudad iberorromana de baitolo/Baetulo.

Palabras clave: Hispania. baitolo. Baetulo. Epigrafía paleohispánica. Cepo de ancla de plomo.

Abstract: The lead stock in the Guerra collection is the first of its category found with an Iberian inscription: baitolo. The most feasible interpretation is to consider it as a place name, either as the name of the city of baitolo/Baetulo, the modern Badalona, which issued coins with the legend baitolo in the $2^{\text {nd }}$ quarter of the $1^{\text {st }} \mathrm{c}$. $\mathrm{BC}$, or as the name of the nearby river, the modern Besós. Greek and Roman inscriptions on stocks basically contain ship-owner names and deity names. In the latter case, they coincide with the name of the ship, which would also allow a place name to be expected on a stock, as geographical ship names are frequent. The ship that carried this anchor was built in the middle of the $1^{\text {st }} \mathrm{c}$. BC and most probably belonged to a Laietanian ship-owner from the Ibero-Roman city of baitolo/Baetulo.

Key words: Hispania. Baitolo. Baetulo. Palaeohispanic Epigraphy. Lead anchor stock.

Recepción: 31.10.2019 | Aceptación: 29.11.2019

Proyecto: Este trabajo ha sido realizado en el marco del proyecto FFI2015-63981-C3-1-P "Hesperia: lenguas, epigrafía y onomástica paleohispánica”, financiado por el Ministerio de Economía y Ciencia de España y "Beyond contacts: tracing identities and cultural change in the Roman West”, financiado por el Social Science and Research Council of Canada a través de una Insight Grant. 


\section{Introducción}

En este trabajo presentamos un ejemplar de gran interés histórico, arqueológico y filológico que actualmente forma parte de la Colección Guerra. Esta colección abarca diferentes períodos históricos y cuenta con más de doscientos objetos de época Clásica (siglos II a.C.-I d.C.). La mayor parte de las piezas de época romana fue adquirida por Esteve Guerra i Marès (1911-1988), que compraba ánforas y otros hallazgos subacuáticos a los pescadores y buceadores que las encontraban con el propósito de salvaguardar el patrimonio local. Esta práctica permite ubicar con cierta fiabilidad muchos de los objetos recuperados en las aguas de la comarca del Alt Empordà. Todas las piezas mencionadas fueron en su día declaradas y catalogadas por el Museu d'Arqueologia de Catalunya y algunas incluso publicadas posteriormente (Martín 2008). Por otro lado, ha sido posible asociar un buen número de los objetos de esta colección a pecios específicos, como los de Cala Bona I (Cadaqués), Punta Blanca (El Port de la Selva), Portaló / Clapera (Cadaqués) y Calella I. ${ }^{1}$ Desafortunadamente, ese no ha sido el caso del cepo que nos ocupa. El hecho de que la colección no haya incorporado piezas nuevas - numismática aparte- durante los últimos cuarenta años proporciona el término ante quem, 1978, para datar el momento en que el cepo entró a formar parte de esta. Los autores pudieron hablar personalmente con algunos de los pescadores anteriormente mencionados, los cuales confirmaron esta hipótesis.

El cepo es una pieza única tanto en el conjunto de cepos de plomo de época grecorromana como en relación con el corpus de inscripciones paleohispánicas en general e ibéricas en particular. Su importancia reside en que sería el primer componente de la arquitectura naval que contiene una inscripción ibérica.

\section{El soporte}

Como se puede observar en la figura 1, se trata de un cepo de plomo que presenta unas dimensiones reducidas: $77 \mathrm{~cm}$ de longitud por $11 \mathrm{~cm}$ de altura y 8 de anchura en la caja central. El peso de la pieza también es modesto $(25,9 \mathrm{~kg})$. Originalmente, debió de formar parte de un ancla de madera de uno o dos brazos. Generalmente, el cepo está unido en ángulo recto al eje de los brazos y la caña, distribuyendo el peso en las cuatro secciones formadas

1 Agradecemos la información a Albert Martín. 
por el eje de cruce de la culata y los brazos, lo que asegura un anclaje eficiente. El cepo se caracteriza por presentar una caja central u 'ojo' colocada alrededor de la caña, de manera que las dimensiones internas de la primera responden a la sección de la segunda. En la caja hay además una barra transversal, prueba de que el cepo fue directamente fundido en la caña después de hacer un agujero en la misma, con lo que se consiguió una unión mucho más resistente entre las dos piezas (Kapitän 1984, 38). Este proceso normalmente se llevaba a cabo vertiendo plomo fundido en un molde cerámico y/o de arena (Haldane 1984, 27; Gargiullo y Okely 1993, 79). El uso de cepos de plomo en anclas de madera se considera una práctica (Haldane 1990, 22) con una amplia cronología (siglos V/IV a.C.-III d.C.) (Haldane 1984, 13; Purpura 2003).

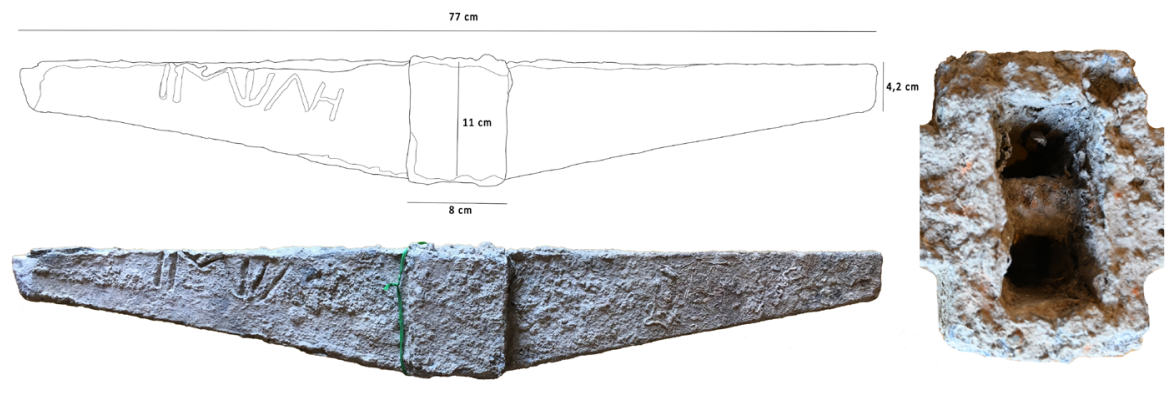

Fig. 1.

Dibujo y fotografía del cepo y sus medidas (elaboración propia).

\section{La inscripción}

En el ancla hay dos inscripciones realizadas en las caras opuestas de los brazos e idénticas entre sí. ${ }^{2}$ Ello permite conjeturar que se elaboraron con la ayuda de una matriz y mediante la técnica de la incisión en frío, puesto que no se aprecian las rebabas características de las inscripciones hechas en caliente; las incisiones son afiladas y rectilíneas, como se aprecia con claridad en los signos ba e i (fig. 2). En tal caso, sería la primera vez que esta técnica se detecta en la epigrafía ibérica y paleohispánica en general. A diferencia del sellado, que se lleva a cabo mediante una matriz incisa que genera una inscripción en positivo, en este caso, la matriz sería en positivo, por lo que el resultado aparece en negativo. Al realizarse en frío, aunque el plomo es dúctil, se tendría que haber aplicado una cierta fuerza. Solo se conoce un sello sobre plomo rea-

2 El código Hesperia de esta inscripción en el Banco de Datos de inscripciones paleohispánicas es el $B D H$ GI.00.01. 
lizado en frío con inscripción ibérica, y precisamente corresponde a un doble sello sobre una etiqueta localizada en baitolo/Baetulo y en la que se menciona a aiuniltun ( $B D H$ B.41.05). Podría encontrarse un posible paralelismo con la inscripción latina del ancla hallada en Blanes, cuya inscripción (SEPTV) presenta características similares (Ripoll 1962). ${ }^{3}$ En general, esta técnica es la habitual en el contramarcado de lingotes de plomo.
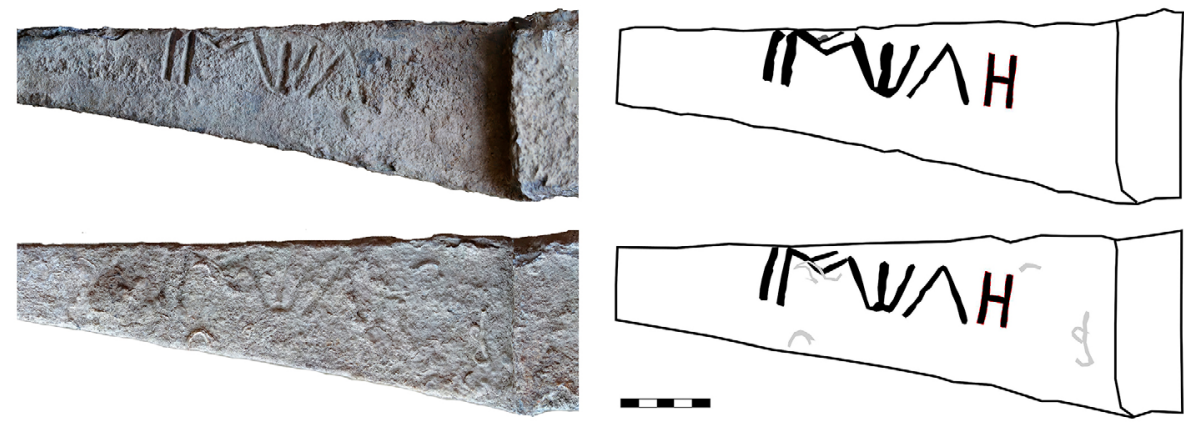

Fig. 2.

Imagen y dibujo de los inscripciones

baitolo (elaboración propia).

El hecho de que la inscripción se elaborase mediante una matriz podría ser indicativo de que se esperaba que fuese utilizada en más de una ocasión, lo que compensaría el esfuerzo realizado en la fabricación de esta. Además, y dejando a un lado que una misma nave pudiese llevar varias anclas, probablemente todas marcadas con la misma inscripción, cabe igualmente la posibilidad de que una misma matriz se usara en diferentes naves del mismo armador. No obstante, el mencionado esfuerzo es insignificante en comparación con el que supone construir una embarcación, por lo que tampoco se puede descartar que fuera un encargo único, específico para una embarcación concreta.

El epígrafe se desarrolla de izquierda a derecha, como es habitual en las inscripciones ibéricas nororientales. Ambas inscripciones se sitúan de forma perfectamente simétrica, el primer signo empieza a $13 \mathrm{~cm}$ del borde, se desarrolla durante $14 \mathrm{~cm}$ y finaliza a $6 \mathrm{~cm}$ de la caja. Dicho primer signo está

3 La inspección confirma que se trata de una técnica similar con desigualdad en la profundidad del trazo, más marcado en la parte superior y a la izquierda, siendo el último trazo del carácter, $\mathrm{V}$, casi inapreciable. No obstante, la inscripción mide únicamente $7 \mathrm{~cm}$ de longitud, la mitad que la de baitolo. Agradecemos las facilidades del Museu Marítim de Barcelona para inspeccionar esta pieza. 
ajustado por su parte superior al lateral superior y puede que incluso perdiera una pequeña porción, puesto que mide $3,2 \mathrm{~cm}$, en tanto que los otros signos presentan una altura uniforme de $3,5 \mathrm{~cm}$ y se desarrollan en diagonal con aproximadamente $8^{\circ}$ de inclinación.

Una de las inscripciones se puede leer con mayor claridad, puesto que en la otra el nivel de las concreciones colmata parcialmente muchos de los trazos. No obstante, en el último signo de ambas inscripciones, las concreciones casi han cubierto los trazos. Esto podría deberse a que, originalmente, el signo final hubiera sido realizado ejerciendo menor presión sobre la matriz, de forma que la profundidad final del trazo sería inferior a la de los otros signos. Los signos restantes parecen presentar una profundidad decreciente, circunstancia que refuerza esta hipótesis y que constituye un fenómeno documentado en los resellos en frío que marcan lingotes de plomo (Colls et al. 1986, 65). Lo anterior podría haber causado que un hipotético sexto signo no apareciera al haber quedado oculto bajo las concreciones, signo para el que habría espacio, teóricamente, entre el signo o y la caja del cepo. Dicho hipotético signo podría explicar la posición de la inscripción, que se habría ajustado entre el borde superior y la caja. No obstante, tal circunstancia solo se podrá verificar una vez restaurada la pieza y eliminadas las concreciones.

Los signos identificados son bal, il, con los trazos superiores cortos y en diagonal, to1, con los trazos exteriores inclinados ligeramente hacia fuera, 11 y o1, aunque la escasa visibilidad del trazo interior impide concretar si es estrictamente horizontal o si presenta alguna inclinación. Estas variantes son características de la escritura ibérica no-dual propia de los siglos II-I a.C. (Ferrer 2005, 969) y, más concretamente, de las leyendas monetales de bronce.

De hecho, la lectura baitolo de ambas inscripciones se corresponde con la de las emisiones monetales de la ciudad de baitolo/Baetulo (Badalona) (BDH Mon-8), en el primer cuarto del s. I a.C. En la paleografía de la leyenda de tales emisiones, todas las variantes de signos son muy similares. No obstante, en la $6^{\mathrm{a}}$ emisión, la distancia entre los signos ba e $\mathbf{i}$ es significativamente mayor $\mathrm{y}$, además, los trazos del signo i se extienden en vertical. En la inscripción del cepo, por el contrario, ambos signos aparecen muy juntos y los trazos del signo i se desarrollan en diagonal. En las emisiones $2^{a}, 5^{a}$ y $7^{a}$, que acuñan cuartos, los trazos verticales de los signos ba e $\mathbf{i}$ aparecen ligeramente inclinados hacia la izquierda, lo que no sucede con la inscripción del cepo. Especialmente en la $1^{\text {a }}$ emisión, representada por sextos - pero también en el resto de las emisiones-, la distancia entre los signos $\mathbf{i}$ y to es significativamente mayor 
que en la inscripción del cepo, donde los signos llegan a tocarse. Así pues, las leyendas de la $3^{\mathrm{a}}$ y $4^{\mathrm{a}}$ emisión serían las más similares a la inscripción del cepo. Sin embargo, el ángulo de abertura de los trazos diagonales del signo i de la inscripción del cepo es significativamente mayor que el de cualquiera de los mismos signos i de las emisiones de baitolo. No es posible establecer que la inscripción del cepo sea exactamente igual que alguna de las variantes documentadas en las emisiones de la leyenda monetal, si bien las diferencias son mínimas y seguramente estuvieron condicionadas por el soporte, un cuño de pocos milímetros en el caso de las monedas o una matriz de $14 \mathrm{~cm}$ en el del cepo.

Como la mayor parte de onomásticos ibéricos, antropónimos, topónimos y teónimos, baitolo está formado por dos elementos, pero la segmentación de estos no es unánime. La propuesta seguida mayoritariamente, la correcta a nuestro entender, es identificar bai y tolo (Faria 1995, 323; Rodríguez 2002, 44, 2005, 61; Ferrer 2013b, 154; 2013a, 142; Orduña 2014, 73; Sabaté 2017, 169; Moncunill y Velaza 2019, 122). Esta propuesta cuenta con abundantes paralelismos; especialmente, en el campo de la toponimia ibérica.

En cambio, para Untermann $(1998,82)$, la segmentación correcta es bait y olo, puesto que identificaba la raíz bait como la misma que figuraría en el río Baetis, el Guadalquivir actual, el nombre antiguo de la actual Béziers, Baeterrae y el frecuente elemento baites (Untermann 1990, 183; Moncunill y Velaza 2019, 120). El segundo componente podría ser el elemento olo(r/ś) (Rodríguez 2014, $\mathrm{n}^{\circ}$ 108), que aparece en olortikirs (F.11.1) y en olośoŕdin (CNH IV 67), en una dracma de imitación. Velaza (2011), por su parte, considera que ambas son posibles, pero sugiere la posibilidad de segmentar bait y ol, o bai y tol, con un posible morfo o, característico de algunos topónimos, como ilduro ( $B D H$ Mon-11). Alternativamente, Jordán 2012-14, 179, plantea para baitolo y para la serie de topónimos ibéricos en -o una posible etimología indoeuropea.

Así pues, de acuerdo con la propuesta mayoritaria, el primer componente de baitolo sería bai, que no aparece explícitamente en los índices de formantes antroponímicos ibéricos (Untermann 1990; Rodríguez 2014), aunque Faria $(1995,323)$ sí lo consideró como tal. En cualquier caso, este elemento aparece claramente representado en otro topónimo, la leyenda monetal eusti(baikula) (BDH Mon-7), donde baikula remitiría asimismo al nombre de la ciudad ausetana Baikov入a (Ptol. 2.6.69), y con los Baeculonenses estipendiarios del convento Tarraconense (Plin. NH 3, 23). Además, el elemento bai podría fi- 
gurar en el posible topónimo ildubaiŕ de la lámina de plomo de La Balaguera (BDH CS.13.07; Ferrer 2013b, 154). En general, la aparición de bai en antropónimos no es especialmente clara, pero, entre los mejores candidatos, estarían baitaś en una lámina de plomo fragmentada de Ullastret (C.2.5) y beŕbai en el plomo monetiforme del Camp de les Lloses ( $B D H$ B.04.01), aunque en ninguno de los dos casos está claro que se trate realmente de antropónimos. Quizá también se podría contar con baiti en un pondus de Azaila (E.1.356), puesto que ti aparece asimismo como formante en antropónimos; tal es el caso de biuŕti en una cerámica de Sant Martí d'Empúries (BDH GI.10.02; Ferrer 2013a, 126). Finalmente, el elemento ibérico bai podría igualmente tener relación con el Bai del teónimo aquitano Baigorixo (Gorrochategui 1984, $\mathrm{n}^{\circ} 468$ ), cuyo primer componente se relaciona con el vasco ibai / 'río', en tanto que el segundo lo hace con gorri/'rojo'. En esta línea, puede que el elemento bai estuviera presente en el posible teónimo banbaibar (Ferrer 2015, 16).

El segundo componente de baitolo es tolo(r), un formante antroponímico conocido (Untermann 1990, $\mathrm{n}^{\circ} 129$; Rodríguez 2014, $\mathrm{n}^{\circ}$ 88) que se documenta claramente en bardastoloŕ (C.17.1) en el plomo de Sant Just Desvern. Por su parte, la forma tolo estaría presente en taŕtolo (C.2.9), en una copa ática de Ullastret (Ferrer y Medina 2018, 129) quizá como antropónimo o como teónimo, puesto que aparece asociado al concepto baiketa, variante de baikar (Ferrer 2019, 54). La misma incertidumbre existe en cuanto a su aparición en una inscripción fragmentada sobre un ánfora de Ensérune que contiene el elemento tolo (B.1.338) además de en un esgrafiado sobre cerámica ática de Pech Maho (B.7.3) cuya lectura final es dudosa, posiblemente toloiger $[\boldsymbol{e}]$ en lugar del tolonkia de $M L H$ II. Dos inscripciones inéditas en las que el elemento tolo reaparece proceden del mismo yacimiento, una de ellas lo hace en la forma toloko (Moncunill 2016, 82), también documentada en una inscripción rupestre de $\operatorname{Er}(B D H$ PYO 05.05). No obstante, su forma latinizada, Toloco, aparece como antropónimo en varias inscripciones latinas, en territorio ibérico o sus inmediaciones (CIL II 3450, 1389 y HEp 15, 368), mientras que lo hace en la forma celtibérica toloku en el tercer bronce de Botorrita (K.1.3). El elemento tolo está asimismo presente en los topónimos Tolobi (Mela 2.5.90), no muy lejos de baitolo/Baetulo, Tolous (It. Ant. 391.3) y Labitolosa (CIL II $3008=5837$ ), ambas en Huesca.

Así pues, la primera hipótesis en cuanto a cómo interpretar el elemento baitolo es considerar que se trata de un topónimo, el nombre de una ciudad o de un río, puesto que es esta interpretación es la que emana directamente de 
su presencia en las fuentes clásicas y epigráficas del s. I d.C., como Baetulo, y en las leyendas monetales del s. I a.C. como baitolo (BDH Mon-8, uid. infra), identificando a la autoridad emisora, que, en el caso ibérico, se corresponde casi siempre con el nombre de la ciudad (Ferrer 2012; Ripollès y Sinner 2019).

Conocemos los topónimos ibéricos gracias a las fuentes clásicas, las fuentes epigráficas latinas y las leyendas monetales ibéricas, en las que mayoritariamente aparecen topónimos y etnónimos basados en topónimos (Ferrer 2012; Ripollès y Sinner 2019). En las inscripciones ibéricas, la presencia de un topónimo previamente clasificado como tal por aparecer en alguna de las fuentes anteriores resulta muy esporádica. Sería el caso del elemento usekerdeku$^{4}$ del mosaico de Caminreal (E.7.1), conocido tanto por las emisiones monetales de la ceca usekerde ( $B D H$ Mon-26), como por las fuentes clásicas

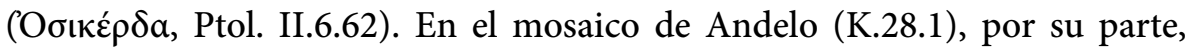
aparece el elemento bilbiliars, correspondiente a una ceca celtibérica, bilbilis (BDH Mon-73), también mencionada por las fuentes clásicas. Quizá sería asimismo el caso de la referencia auśes en una placa de piedra procedente de Empúries (BDH GI 10.07), la cual podría hacer referencia a la Aü $\sigma \alpha$ de las fuentes clásicas (Ptol. II.6.69) y de la epigrafía latina (CIL II 6110, Ausone) y que emite moneda como auśesken ( $B D H$ Mon-73). En cambio, la propuesta de Untermann (1990, D.8.1) de identificar alaun (BDH Mon-16) en la inscripción de la Roca dels Moros (D.8.1; El Cogul) es una mala lectura (Silgo 1994, 34). En los tres casos, su posición en la inscripción y los morfos que los acompañan permiten también su interpretación como topónimos indicando la procedencia de los personajes principales. La aparición de baitolo en el cepo de plomo de la Colección Guerra se añade ahora a esta breve lista de excepciones de topónimos conocidos por leyendas monetales que aparecen fuera del contexto monetal.

La posibilidad de que se trate de un antropónimo es teóricamente factible, pero no probable, debido a la poca productividad de los formantes bai y tolo en la formación de antropónimos, circunstancia extrapolable a la segmentación alternativa bait y olo (Untermann 1998). Además, como se ha indicado anteriormente, en los tres casos en que los topónimos de las leyen-

4 La transcripción de usekerde con la dental sonora, a pesar de que no se trata de una ins-

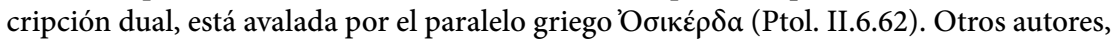
ante la duda de cuál sería la transcripción correcta, prefieren transcribir las oclusivas dentales y velares de las inscripciones no-duales de forma general, pero arbitraria, siempre como sordas: usekerte. En el mismo caso se encuentran las referencias a ilduro e ildiŕda con paralelos que avalan el uso de la sonora, Ferrer 2005, 957, n. 1 y 4. 
das monetales han aparecido en inscripciones no monetales, su función como topónimos era clara. Por ello, no parece probable que baitolo funcione como antropónimo en esta ocasión, aunque es plausible suponer que esta práctica hubiera existido. No obstante, hasta donde podemos documentar en ibérico, aunque topónimos y antropónimos comparten formantes, forman conjuntos disjuntos una vez combinados.

Cabe igualmente la posibilidad de que se tratase de una divinidad, pero no existen argumentos de peso que lo demuestren excepto que, al ser baitolo también el nombre de un río, pudiera tener asociada una divinidad homónima. Este es el caso de los Potamoi griegos, hijos de Océano y Tetis, considerados dioses fluviales que eran personificaciones de ríos, como el Éufrates (Hes. Th. 337-345). Asociar divinidades con ríos cuenta con paralelismos en la Hispania indoeuropea (Olivares 2000). Con todo, no ha podido confirmarse que esta práctica fuera común entre los iberos. De hecho, las divinidades ibéricas son muy escasamente mencionadas en las inscripciones latinas, pero se puede apreciar que igualmente responden a la estructura bimembre de antropónimos y topónimos. El inicialmente leído Seitundo de Susqueda (IRC V, p. 83) es, en realidad, Sertundo (Vidal 2016, 198), formado por seŕtun y do. El segundo, Betatun (Corzo et al. 2007), estaría formado por bete y atun (Ferrer et al. 2018, 182). Por último, el tercero, Salaeco (AE 2010, 754), podría estar formado per śalai y por ko (Velaza 2015). Recientemente, el estudio de las inscripciones rupestres ha ampliado la nómina de posibles divinidades ibéricas, de forma que ya es posible empezar a hablar de un panteón ibérico (Ferrer 2019, 54), entre otros: urdal, balkar, garde, tikanal, artiunan, teleuś, okal y śauś.

\section{Los cepos inscritos griegos y latinos}

En la bibliografía especializada (Gianfrotta 1980; Hesnard y Gianfrotta 1989; Gianfrotta 1994; Fenet 2016; Gianfrotta 2017) hemos identificado cerca de 90 inscripciones sobre cepos de plomo. ${ }^{5}$ El significado de dichas inscripciones ofrece dos alternativas principales: antropónimos (59 \%) y teónimos (39\%), además de dos referencias a legiones. Todas las inscripciones griegas contienen teónimos, cuyo empleo se reduce a menos del $20 \%$ en las latinas (fig. 3).

5 No hemos tenido en cuenta las anclas de piedra, algunas de las cuales también presentan inscripciones y tampoco las que solo contienen numerales o decoraciones, Fenet 2016, 573 y 596 . 
Así pues, el caso más habitual -especialmente en inscripciones latinases encontrar en los cepos de las anclas un antropónimo ${ }^{6}$ que casi siempre se interpreta como el nombre del armador de la nave. Un buen ejemplo de lo anterior es L. Ferranius Celler (A15) en Populonia, que también aparece en otro pecio en Foce Verde (Latina) y en un tercero en Cullera (Valencia), uno de los escasos ejemplos en que el nombre del mismo armador aparece en diversos pecios. Normalmente la inscripción solo figura sobre uno de los cuatro laterales del cepo, siendo el lateral más ancho la opción preferida, aunque a veces se utiliza el más estrecho, como en el caso de C. Aquilli Proculli (fig. 3.4) en Castelabate (A7), en la que además aparece por duplicado.

La segunda casuística más frecuente es encontrar el nombre de una divinidad y/o un epíteto que la caracteriza. ${ }^{7}$ Entre las inscripciones griegas, encontramos ejemplos de ello en "Hpa (Fig. 3.1) en Reggio Calabria (I19), "H $\mathrm{\rho} \mu \eta$ (fig. 3.5) en Belvedere, ${ }^{8} \mathrm{H} \rho \alpha \kappa \lambda \tilde{\eta} \varsigma$ en Capo Zafferano (Palermo) (I25) y $\Sigma \omega ́ \tau \varepsilon ı \rho \alpha ~(f i g . ~ 3.3)$, referida a 'A $\theta \eta v \tilde{a}$ en Monasterace (Reggio Calabria) (Gianfrotta 2017) y otras seis localizaciones (I10-I15). Y entre las latinas: Vesta en Capo Vaticano (Calabria) (I17), Venus en Maratea (I9), Saluia en Ventotene (Latina) (I16), etc. A veces en forma de doble dedicación, como Ceres / Isis en Nora (I22). Finalmente, una inscripción recientemente aparecida en Messina (Roca y Oliveri 2016, 246) contendría un mensaje explícito respecto del objetivo del ancla, Salutem (fig. 3.2).

Cuando el nombre que figura en el ancla se corresponde con el de una divinidad, se considera que también sería el de la nave (Gianfrotta 1980, 109; 2017, 254; Fenet 2016, 320). Las inscripciones del siglo IV a.C. correspondientes a la flota militar ateniense documentan casi 300 nombres de naves que responden a epítetos de dioses y heroínas: Thetis; nombres descriptivos: Prote; ideas abstractas: Demokratia; nombres de objetos y animales: Panthera. Casson $(1995,353)$ estima que el $10 \%$ del total de nombres griegos de naves eran nombres de origen geográfico: Ionike, Hellas, Europe, Crete, Naukratis, etc. Tal sería el caso del navío Syracusa, famoso por sus grandes dimensiones (Casson 1995, 359). Los romanos no siguieron esta pauta y redujeron considerablemente la variedad de conceptos. Así, gran parte de los navíos fueron bautizados con nombres de divinidades, como Minerva, Mercurius, Pietas, Prouidentia, etc. Y de ríos mayoritariamente cuando se trata de nombres de

6 El código entre paréntesis hace referencia al catálogo de Hesnard y Gianfrotta 1989.

7 El código entre paréntesis hace referencia al catálogo de Fenet 2016.

8 http://www.museodeibrettiiedelmare.it/it/guida/androne-del-palazzo/. 
Baitolo, una doble inscripción ibérica en un cepo de ancla de plomo del siglo I a.C.

origen geográfico, que también podrían hacer referencia a la divinidad homónima, como Danubius, Nilus, Tigris, Eufrates, Padus, Rhenus y Tiberis (Casson 1995, 358-359). De hecho, algunos de los nombres documentados en anclas figuran en la relación de nombres de naves militares pertenecientes a la flota de Miseno (Gianfrotta 1980, 110), como Vesta, Venus, Saluia, Ceres e Isis, tanto en trirremes como en cuadrirremes.

1

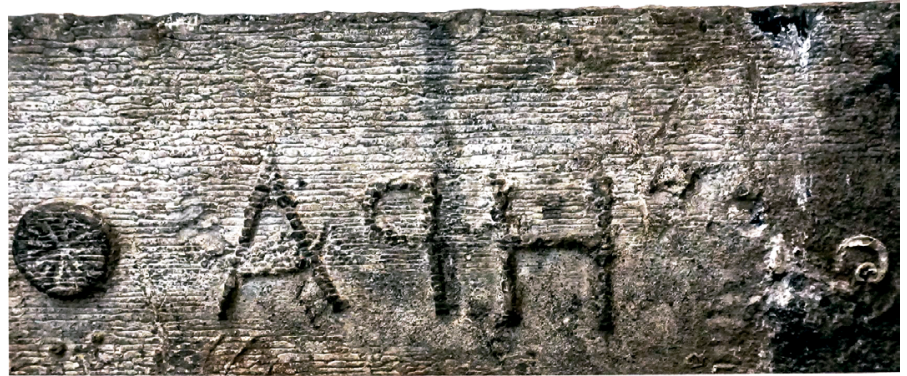

2

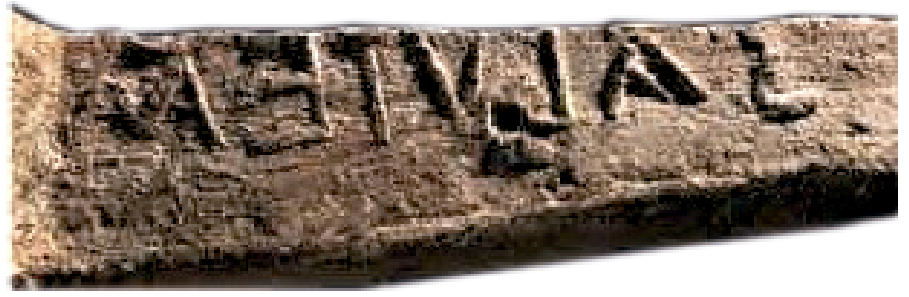

3
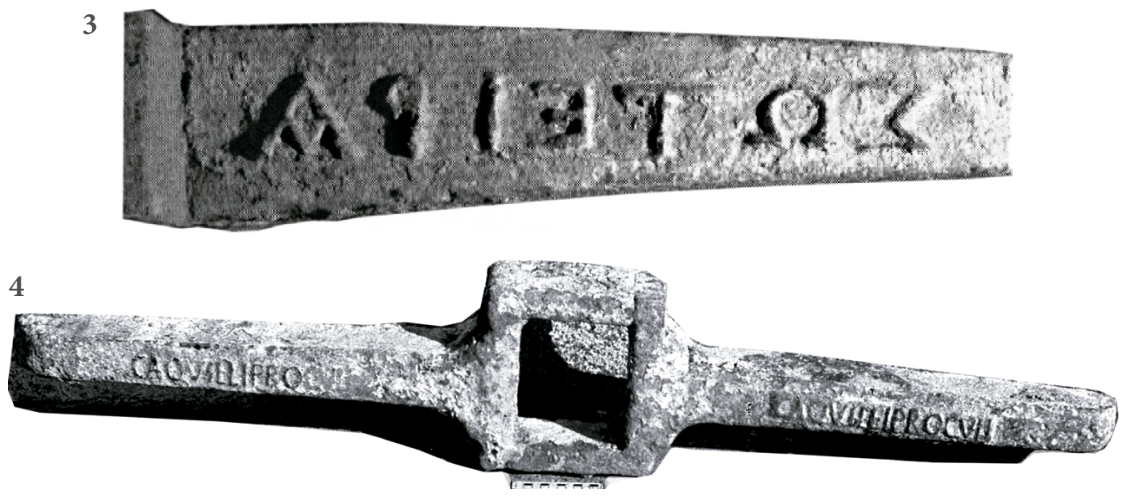

5

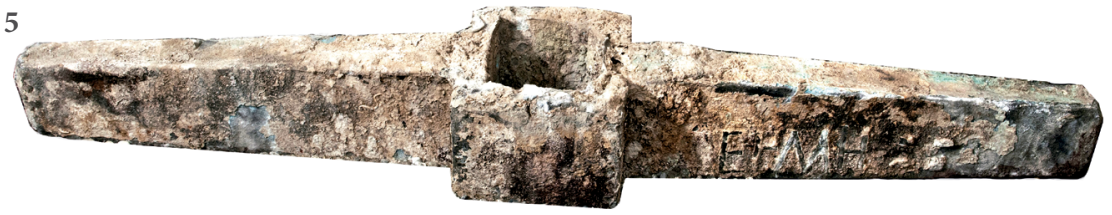

Fig. 3. Ejemplos de cepos de plomo inscritos griegos y latinos. 1: "Hpa (Museo Archeologico

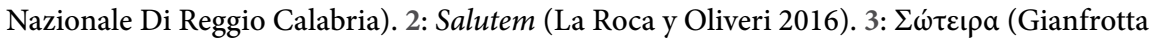
1994). 4: C. Aquilli Proculli (Gianfrotta 1994). 5: "H $\rho \mu \eta$ (Museo del Mare, Cetraro). 


\section{Consideraciones sobre su autenticidad}

Teniendo en cuenta que se trata de una pieza única y sin parangón dentro del corpus de epigrafía ibérica y que la inscripción no procede de una excavación arqueológica (subacuática) regular, sino de una colección privada, es necesario considerar la posibilidad de que se trate de una falsificación. Aun así, cabe recordar que la práctica totalidad de cepos de plomo conocidos proceden de hallazgos aislados y descontextualizados.

La presencia de una leyenda monetal en un objeto que no sea una moneda suscita de inmediato una sospecha sobre su autenticidad, porque tales leyendas suelen ser el primer texto que un falsificador tiene a mano y porque, además, son textos cortos y relativamente sencillos de copiar. Disponemos de múltiples falsificaciones en las que el texto se limita a copiar algunas de las leyendas monetales más conocidas, como ildiŕda ( $B D H$ Mon-18), en un sillar de arenisca de la Ermita de San Salvador (Les Borges Blanques) (Garcés y González 2018, 339), ildiŕda ( $B D H$ Mon-18) y kese (BDH Mon-12) en un prisma calcáreo de Monteró (Camarasa; Garcés y González 2018, 343), śaiti (BDH Mon-35) en el borde de un kalathos del Tossal del Metxut (Almenar) (Garcés y González 2018, 345) y kelse (BDH Mon-21), baśti (BDH Mon-29) y orósis (BDH Mon-86) en los glandes de plomo de la colección de Figuerola del Camp (Ferrer 2017, 98).

No obstante, si los soportes se clasifican en función de la dificultad de su falsificación, los cepos de plomo estarían en el extremo opuesto, ya que son probablemente uno de los objetos más difíciles de falsificar o incluso de reproducir (Quero 2009). Si bien el cepo que nos ocupa posee unas dimensiones relativamente discretas dentro del corpus de cepos conocidos, su peso y dimensiones complican extremadamente su falsificación. Además, el proceso de fabricación de un cepo requiere elaborar previamente un molde, cerámico o de madera, sobre el que se vierte el plomo fundido. Al tratarse de un cepo fijo, además, sería necesario colocar la caña de madera del ancla con el orificio en dicho molde de manera que el cepo encaje perfectamente y forme la barra transversal que atraviesa la caja. Por si esto no fuese suficientemente complejo, el cepo presenta claras concreciones de fauna y flora marina, algunas de ellas por encima de los signos, síntoma inequívoco de que ha pasado algunos años sumergido en el mar. Por último, el grado de concreción que ambas caras presentan es diverso, seguramente por estar una de ellas protegida por la arena. 
Tampoco parece viable pensar en un cepo auténtico sobre el que se hubiera realizado una inscripción falsa, puesto que eso requeriría limpiar las concreciones originales, realizar la falsificación (por partida doble) y volver a depositar el cepo en el mar durante algunos años para que una nueva capa uniforme de concreciones se genere.

Adicionalmente, resulta significativo el hecho de que ambas inscripciones fueran realizadas en frío con algún tipo de matriz, dado que ello requiere el esfuerzo adicional de fabricar dicha matriz. Que la inscripción sea doble también es algo poco frecuente, y no encaja con el objetivo de un falsificador. La repetición del mismo epígrafe no supone ningún valor de mercado añadido a la pieza. Por otro lado, siguiendo con la hipótesis de la falsificación, una simple inscripción elaborada sin la ayuda de una matriz habría sido suficiente para incrementar el valor del cepo con mucho menos esfuerzo. Tampoco la técnica de realización, la incisión en frío es la más habitual, puesto que, normalmente, las inscripciones sobre objetos de plomo de gran tamaño y peso - especialmente cepos y lingotes - son el resultado directo del proceso de fabricación de la pieza. Tales inscripciones se graban en negativo en el molde cerámico en el que el objeto se fundirá posteriormente, gracias a lo que la inscripción aparece en relieve. Si se construye un molde para elaborar un cepo falso, resultaría mucho más sencillo utilizar esta técnica que no seguir el laborioso procedimiento de grabar a posteriori el epígrafe por duplicado ayudándose de una matriz que también hay que fabricar.

Finalmente, no parece que el hecho de que el cepo de plomo llevara una inscripción fuese un dato significativo para los propietarios del objeto, dato que no hicieron constar en el catálogo ni en la exposición de la pieza, y tampoco lo comunicaron a los diversos investigadores que visitaron la colección ni en las diversas entrevistas concedidas a medios de comunicación locales y provinciales. Lo cierto es que la inscripción fue identificada de forma casual por uno de los autores del presente trabajo en julio de 2019 durante una inspección de otros materiales de la colección.

Resumiendo, a pesar de tratarse de un texto muy simple y fácilmente falsificable - una leyenda monetal-, el soporte - un cepo de plomo- es extremadamente complejo de falsificar. Esto se une a la fecha aproximada en que calculamos la pieza fue adquirida (la década de 1970), momento en que era mucho más sencillo recuperar una pieza del fondo marino que falsificarla. Finalmente, ningún objeto falso ha sido documentado en la Colección Guerra hasta la fecha. Todo lo anterior nos lleva a considerar que se trata de una inscripción auténtica casi con total seguridad. 


\section{Contexto epigráfico y arqueológico: la ciudad de baitolo / Baetulo}

El puerto base de la nave que estaba equipada con el ancla objeto de este trabajo muy probablemente fue la ciudad ibero-romana de baitolo/Baetulo. Las fuentes clásicas del s. I d.C. identifican a Baetulo, actual Badalona y adaptación latina de baitolo, como el nombre de una ciudad situada entre Barcino (Barcelona) e Iluro (Mataró) (Plin. NH 3.22; Ptol. 2.16.18; Mela 2.90), pero también como el de un pequeño río cercano - el Besós-, que sería probablemente en origen el que habría dado nombre a la ciudad. Los antecedentes de baitolo/Baetulo posiblemente deben buscarse en el cercano oppidum ibérico del Turó d’en Boscà, a escasos dos kilómetros hacia el interior.

No obstante, las emisiones de la ceca baitolo ( $B D H$ Mon-8) se sitúan ya en la ciudad romana (Padrós 2012, 44). Ahora bien, preferimos la denominación iberorromana puesto que, en el momento de su fundación, casi todos los pobladores de la nueva ciudad del Turó d'en Rosés, aún baitolo según sus emisiones monetales, eran íberos. Este hecho puede acreditarse a partir de la epigrafía, dado que la única documentación epigráfica identificada en el momento fundacional y en gran parte del siglo I a.C. en época republicana en baitolo es ibérica (Torra 2009). Además, muy probablemente también las élites dirigentes eran íberas, ${ }^{9}$ y serían las responsables de la emisión de las monedas de bronce de baitolo con leyenda e iconografía ibérica (Sinner y Ferrer 2016; Amela 2018). Por esta razón, utilizamos la doble denominación baitolo/ Baetulo para referirnos a la ciudad del Turó d'en Rosés. ${ }^{10}$ Las referencias a Baetulo, tanto de las fuentes antiguas, como de las epigráficas más antiguas, son del siglo I d.C. avanzado. Así, los baetulonenses son citados en una inscripción del 98 d.C. (IRC I 139).

La fecha de abandono del poblado ibérico del Turó d’en Boscà se sitúa alrededor del 100 a.C., aun cuando se detecta alguna ocupación puntual hasta el 90 a. C. (Zamora 1995; Padrós 2005, 524), mientras que la fundación de la ciudad ibero-romana del Turó d’en Rosés se produjo en algún momento del

9 Como ocurre con los cuatorviros de Iulia Libyca (Ferrer et al. 2018), puesto que tres de ellos aún ostentan nombres ibéricos en época augustal, mientras que el cuarto, que ya lleva nombre romano, también es de filiación ibérica: Bella $:$ Gaisco $: f($ ilius $) / \underline{B}$ ella : Bastobles : f(ilius) / Adinildir : Betepe $[-\cdot f($ ilius $)] /$ Corneli $:$ Erdoild[ir $\cdot f($ ilius $)] /$ scriptum . est $\cdot$ IIII $\cdot$ viratum.

10 En la ciudad vecina ilduro/Iluro encontramos un buen paralelo, Sinner y Ferrer $2016 \mathrm{y}$ 2018; García 2017, 75-89. 
primer cuarto del siglo I a.C., aunque la fecha precisa sigue siendo tema de debate, existe un cierto consenso en situarla entre los decenios 90/80 y 80/70 a.C. (Padrós 2012, 49). No obstante, con anterioridad a la fundación de la ciudad, ya existió un suburbium en Illa Fradera, con una cronología fundacional del primer cuarto del siglo I a.C. y en el que las estructuras, de filiación itálica, ya se orientan siguiendo el futuro trazado de la ciudad. Posteriormente, son substituidas por un centro de producción de ánforas Pascual 1 a mediados del s. I a.C. (Antequera et al. 2010; Padrós 2012, 51).

Además, gracias a los centros de producción de ánforas situados en su entorno inmediato, como el de Can Peixau — vinculado a la marca M. Porci-, el existente en Illa Fradera o el recientemente excavado de La Estrella, ${ }^{11}$ baitolo/ Baetulo probablemente actuó como centro de distribución de la producción de las villas de su hinterland (Comas y Carreras 2008, 185). Los estudios arqueométricos realizados en los pecios Cap del Vol (Vivar et al. 2013, nota 5), Els Ullastres y Port-Vendres 4 y 5 (Martínez 2016, 146) confirman que una gran parte de las ánforas que estas naves transportaban procedían de los centros productores de baitolo/Baetulo. Esta actividad distribuidora requería la existencia de una zona portuaria de la que se han hallado indicios al suroeste de la ciudad, donde aparecieron unas grandes piedras en pendiente, muy rodadas, que se interpretaron como una zona de carga/descarga de mercancías (Comas 1998, 221). Adicionalmente, dos grandes depósitos de ánforas y tres dolia, combinación característica de las zonas portuarias, han sido asimismo documentados (Comas y Padrós 2008, 83). También se considera que el propio estuario del Besós sirvió como puerto complementario al de la ciudad (Izquierdo 2009, 185).

Las monedas de la ceca de baitolo se estructuran, según los últimos estudios (Padrós 2002, 111; 2012, 53; Amela 2018), en siete emisiones distribuidas durante el primer cuarto del s. I a.C. Además, en Illa Fradera se localizaron tres plomos monetiformes que imitan la iconografía de los sextantes de la $1 .^{a}$ emisión con delfín y marca de valor be II en el reverso, que, aunque se ha propuesto interpretar como ponderales (Padrós 2012, 53), parece que simplemente constituyeron una alternativa a los divisores de bronce en momentos de carestía (Ferrer 2014, 63; Amela 2018, 90).

11 Donde se han documentado instalaciones de procesado de uva (prensas, canales, lacus), varias marcas de M. Porci y, sobre todo, una inscripción lapidaria con el nombre Porci que parece indicar que se trata de una de las fincas de este personaje. 
Hasta la aparición del ancla objeto de este trabajo, la mejor evidencia de la existencia de armadores indígenas en la Hispania Citerior en época tardo-republicana también estaba relacionada con baitolo/Baetulo. Se trata del pecio del Cap del Vol (Nieto 1982; Vivar et al. 2013) de unos 15 metros de eslora, localizado en el Port de la Selva y que, según sus excavadores, presenta técnicas de construcción de tradición indígena. Su cargamento principal consistía en entre 200 y 400 ánforas Pascual 1, algunas de ellas con los sellos M. Porci y Iuli Teophil, y algunas producidas en baitolo/Baetulo, por lo que se ha interpretado como un pecio fletado en baitolo/Baetulo con dirección a Narbona. Su hundimiento ha sido fechado entre los últimos años del siglo I a.C. y primeros del siglo I d.C. (Nieto 1982, 168). Otro pecio interesante es el Cala Cativa I, de unos 10 metros de eslora, que muestra la misma tradición constructiva documentada en el Cap del Vol y también se localiza en el Port de la Selva. Cronológicamente, parece que el pecio puede datarse entre los años 40 y 30 del siglo I a.C. y cargaba principalmente ánforas Pascual 1, entre 150 y 200 ejemplares, de las que se desconoce su procedencia.

\section{Conclusiones}

Nos encontramos ante el primer cepo de ancla con una inscripción ibérica hallado hasta el momento. Esta circunstancia es relevante desde el punto de vista de la propia epigrafía ibérica al añadir un nuevo soporte y también una nueva técnica de escritura, y desde la perspectiva del estudio del comercio marítimo en el Mediterráneo occidental en la antigüedad; particularmente, del comercio de vino tarraconense durante el s. I a.C. ${ }^{12}$

La interpretación más probable del epígrafe baitolo es que sea un topónimo, el nombre de una ciudad o de un río, como ocurre en las leyendas monetales de baitolo y en las fuentes clásicas, que mencionan tanto a la ciudad como al río Baetulo. La posibilidad de que baitolo sea un antropónimo es muy poco probable dada la escasa productividad de los formantes bai y tolo en la formación de antropónimos y debido al hecho que, en los tres casos en que los topónimos de las leyendas monetales han aparecido en inscripciones no monetales, su función como topónimos era clara. También podría tratarse del nombre de una divinidad, pero no existen argumentos de peso que apoyen esta hipótesis excepto que baitolo al ser también el nombre de un río, dicho nombre estuviera asociado con una divinidad homónima.

12 Un análisis más detallado de las implicaciones de este hallazgo en el comercio del vino layetano del s. I a.C. en Sinner y Ferrer e.p. 
El conjunto de cepos de plomo inscritos ofrece dos interpretaciones principales: antropónimos y teónimos. En la mayor parte de los casos, el antropónimo se interpreta como el armador, si bien puede coincidir con el propietario de la carga en ocasiones, y solo puntualmente como el fabricante del cepo. Consideramos muy improbable esta última opción en el caso que nos ocupa tanto por la técnica de realización -indicativa de que la inscripción fue posterior a la fabricación - como por la ausencia del elemento característico de las marcas de producción en ibérico: egiar. Puesto que las posibilidades de interpretar baitolo como antropónimo son escasas, la única alternativa que permitiría interpretar baitolo como identificador del armador sería que esta función la asumiera la misma autoridad que emitió moneda bajo la denominación baitolo. No obstante, esta posibilidad implicaría que la ciudad de baitolo/Baetulo participaba, como entidad pública, en actividades comerciales y, actualmente, no hay ningún dato que respalde dicha hipótesis.

Cuando en el ancla aparece un teónimo, este se interpreta como una invocación a la divinidad para que interceda en favor de la embarcación, pero se admite que normalmente coincide con el nombre de la nave y existen diversos paralelismos entre los teónimos documentados en cepos y los nombres de naves conocidos. Esta última alternativa permitiría esperar un topónimo en un cepo. Aproximadamente el $10 \%$ de los nombres de naves griegas tienen nombre de origen geográfico, como el famoso Syracusa. Además, las naves con nombres de río están ampliamente documentadas entre los romanos; por ejemplo, Nilus. En este caso, por tanto, un armador anónimo habría grabado el nombre de la nave sobre el cepo en honor al río, a su posible divinidad homónima o a la ciudad que sería su base operativa y tal vez también el astillero donde se construyó.

Respecto a la cronología, la derivada de la tipología del ancla es demasiado amplia, por lo que no proporciona pista alguna sobre el momento en que la inscripción fue realizada. En cambio, la paleografía de los signos nos sitúa dentro del marco general de la escritura no-dual de los siglos II-I a.C. El paralelismo con el inicio de las emisiones monetales de baitolo y las primeras construcciones previas a la fundación de la ciudad permiten proponer una fecha post quem de principios del s. I a.C. En cuanto a la fecha ante quem, se puede establecer la genérica de finales del s. I a.C. para las inscripciones ibéricas, ya que empiezan a ser esporádicas en la segunda mitad del s. I a.C. No obstante, precisamente en baitolo/Baetulo se ha documentado una de las más modernas, la única segura sobre terra sigillata Itálica, de la forma Goudineau 1, 
del último cuarto del s. I a.C. Así pues, la cronología genérica propuesta es del s. I a.C., aunque teniendo en cuenta que las evidencias más antiguas de la exportación de vino en baitolo/Baetulo corresponden a mediados del s. I a.C., ésta sería la cronología más probable para la construcción de la nave.

Finalmente, el ancla de la Colección Guerra con doble leyenda ibérica baitolo confirma epigráficamente la participación de íberos en el transporte marítimo del vino layetano del siglo I a.C. Esto es algo que ya se suponía arqueológicamente hablando gracias a los resultados obtenidos en la excavación de los pecios de Cap del Vol y Cala Cativa I, por lo que ya podemos hablar con propiedad de armadores íberos. En particular, creemos que nuestro armador era probablemente un layetano de baitolo/Baetulo. En cualquier caso, confirma el papel de la ciudad de baitolo/Baetulo, actual Badalona, y del entorno del río con el mismo nombre, actual Besós, como uno de los principales núcleos operativos en el comercio del vino layetano.

Agradecimientos: Quisiéramos agradecer al Sr. Salvador Guerra que nos haya dejado visitar su colección en varias ocasiones, así como las facilidades y el excelente trato que nos dispensó en cada una de dichas visitas, sin su amabilidad y colaboración este artículo no habría sido posible, así como a A. Martín (arqueólogo municipal de Cabrera de Mar) su inestimable colaboración durante el transcurso de nuestra investigación. También deseamos mostrar nuestra gratitud a V. Revilla (Universitat de Barcelona), J. Velaza (Universitat de Barcelona), C. Carreras (Universitat Autònoma de Barcelona), P. Padrós (Museu de Badalona), R. Geli (CASC), G. Vivar (CASC), C. de Juan (Universitat de València) y V. Sabaté (Universitat de Barcelona) por sus comentarios y sugerencias.

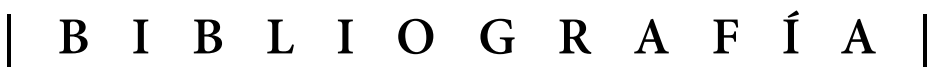

Amela 2018: L. Amela, "baitolo", Varia Nummorum 8, 2018, 85-97.

Antequera et al. 2010: F. Antequera, P. Padrós, A. Rigo y D. Vázquez, "El suburbium occidental de Baetulo", en: D. Vaquerizo (ed.), Las áreas suburbanas en la ciudad histórica. Topografía, usos, función, Córdoba 2010, 173-210.

Casson 1995: L. Casson, Sea and Seamanship in the Ancient World, Baltimore 1995.

Colls et al. 1986: D. Colls, C. Domergue y V. Guerrero, "Les lingots de plomb de l'épave Cabrera 5 (île de Cabrera, Baléares)", Archaeonautica 6, 1986, 31-80.

Comas 1998: M. Comas, "La producció i el comerç del vi a Baetulo. Estat de la questió", en: El vi a l'Antiguitat, economia, producció i comerç al Mediterrani Occidental, Badalona 1998, 219-232.

Comas y Carreras 2008: M. Comas y C. Carreras, "Les àmfores de la laietània: estat de la qüestió", en: Monografies MAC-Barcelona 8, 2008, 177-188. 
Comas y Padrós 2008: M. Comas y P. Padrós: “Deux grands dépotoirs d’amphores léétaniennes, bétiques et gauloises hors les murs de la ville de Baetulo (Badalona)," en: SFECAG, Actes du Congrès de L'Escala-Empúries 2006, Ampurias 2008, 75-86

Corzo et al. 2007: S. Corzo, M. Pastor, A. U. Stylow y J. Untermann, “Betatun: La primera divinidad ibérica identificada", PalHisp 7, 2007, 251-262.

Faria 1995: A. M. De Faria, “Algumas notas de onomástica ibérica”, Portugália 16, 1995, 323 330.

Fenet 2016: A. Fenet, Les dieux olympiens et la mer. Espaces et pratiques cultuelles, Roma 2016.

Ferrer 2005: J. Ferrer i Jané, "Novetats sobre el sistema dual de diferenciació gràfica de les oclusives", PalHisp 5, 2005, 957-982.

Ferrer 2012: J. Ferrer i Jané, "La lengua de las leyendas monetales ibéricas", en: A. G. Sinner, (ed.), La moneda de los íberos. Ilturo y los talleres layetanos, Barcelona 2012, 28-87.

Ferrer 2013a: J. Ferrer i Jané, "Los problemas de la hipótesis de la lengua ibérica como lengua vehicular", ELEA 13, 2013, 116-157.

Ferrer 2013b: J. Ferrer i Jané, "Nova lectura dels ploms ibèrics de La Balaguera (La Pobla de Tornesa): un nou plom explícitament dual", QPAC 31, 2013, 149-157.

Ferrer 2014: J. Ferrer i Jané, "A propòsit d'un sisè de bronze de baitolo amb la llegenda be II", Acta Numismàtica 44, 2014, 57-69.

Ferrer 2015: J. Ferrer i Jané, "Le nouveau corpus d'inscriptions ibériques rupestres de la Cerdagne: deuxième parution”, Sources-Les cahiers de l'Âne Rouge 3, 2015, 7-22.

Ferrer 2017: J. Ferrer i Jané, "Dos vasos caliciformes amb inscripció ibérica ante cocturam del Museu de Figuerola del Camp i el seu espectacular conjunt de falsos", Saguntum 49, 2017, 85-101.

Ferrer 2019: J. Ferrer i Jané, “Construint el panteó ibèric amb l'ajut de les inscripcions ibèriques rupestres”, Ker 13, 2019, 42-57.

Ferrer y Medina 2018: J. Ferrer i Jané y E. Medina, “Una nova inscripció ibèrica procedent de Can Vallmanya (Vidreres): Baika i Baiketa també són Baikar”, Cypsela 21, 2018, 119133.

Ferrer et al. 2018: J. Ferrer i Jané, O. Olesti y J. Velaza, “Nuevas inscripciones rupestres latinas de Oceja y los IIIIviri ibéricos de Iulia Lybica.” DHA 44.1, 2018, 169-195.

García 2017: J. García, Gènesi, fundació i període republicà de la ciutat romana d'Iluro (Hispania Citerior), Mataró 2017.

Garcés y González 2018: I. Garcés y J. R. González, “Inscripciones ibéricas falsas y popularización de epígrafes monetales en la zona de Lleida", Anuari De Filologia. Antiqua et Mediaevalia $8,2018,335-351$.

Gargiullo y Okely 1993: S. Gargiullo y E. Okely, Atlante archaeologico dei mari d'Italia vol II, Roma 1993.

Gianfrotta 1980: P. A. Gianfrotta, "Ancore romane: nuovi materiali per lo studio dei traffici marittimi”, en: J. H. D'Arms y E. C. Kopff (eds.), The Seaborne Commerce in Ancient Rome: studies in archaeology and history, Roma, 1980, 103-116.

Gianfrotta 1994: P. A. Gianfrotta, "Notte di epigrafia marittima. Aggiornamenti su tappi d'anfora, ceppi d'ancora e altro", en: Epigrafia della produzione e della distribuzione, Roma 1994, 591-608.

Gianfrotta 2017: P. A. Gianfrotta, "Fenet A., Les dieux olympiens et la mer. Espaces et pratiques cultuelles", ArchClass 68, 2017, 649-656.

Gorrochategui 1984: J. Gorrochategui, Onomástica indígena de Aquitania, Bilbao 1984. 
Joan Ferrer i Jané | Alejandro G. Sinner

Haldane 1984: D. Haldane, The Wooden Anchor. Tesis Doctoral, Texas A\&M University 1984.

Haldane 1990: D. Haldane, "Anchors of Antiquity", Biblical Archaeologist 53.1, 1990, 19-24.

Hesnard y Gianfrotta 1989: A. Hesnard y P. A. Gianfrotta, "Les bouchons d'amphore en pouzzolane", en: Anfore romane e storia economica: un decennio di ricerche (Siena 1986), Roma 1989, 393-441.

Izquierdo 2009: P. Izquierdo, "Els ports del litoral tarraconense i el seu paper en el comerç del ví", en: El vi tarraconense i laietà: ahir i avui, Tarragona 2007, 179-191.

Izquierdo 2018: P. Izquierdo, “Condiciones y conocimientos náutico-marinas en la antigüedad”, en: J. M. López y M. M. Ros (eds.), Navegar el Mediterráneo, Mazarrón 2018, 17-35

Jordán 2012-14: C. Jordán, "Sobre los topónimos terminados en -o de algunas leyendas monetales ibéricas levantinas", Faventia 34-36, 2012-14, 177-188.

Kapitän 1984: G. Kapitän, “Ancient anchors-technology and classification”, International Journal of Nautical Archaeology and Underwater Exploration 13.1, 1984, 33-44.

La Roca y Oliveri 2016: R. La Roca y F. Oliveri, "Le ancore di salvezza", en: Mirabilia maris. Tesori dai mari di Sicilia, Palermo 2016, 243-245.

Martín 2008: A. Martín Menéndez, "Àmfores Tarraconenses i Bètiques en els derelictes de mitjan de segle I a.C. a la costa catalana", en: SFECAG, Actes du congrés de L'EscalaEmpúries, Ampurias 2008, 103-127.

Martínez 2016: V. Martínez Ferreras, "Las ánforas vinarias de la Layetania. Dinámicas de producción y difusión comercial en el siglo I a.C. y I d.C.”, en: R. Járrega y P. Berni (eds.), Amphorae Ex Hispania: Paisajes de producción y consumo, Tarragona 2016, 139-153.

Moncunill 2016: N. Moncunill, "Novecientos antropónimos ibéricos”, PalHisp 16, 2016, 81-94.

Moncunill y Velaza 2019: N. Moncunill y J. Velaza, Monumenta Linguarum Hispanicarum Band V.2 Lexikon der iberischen Inschriften, Wiesbaden 2019.

Nieto 1982: X. Nieto, "El pecio romano del Cap del Vol. Nuevas aportaciones", Cypsela 4, 1982, 165-168.

Olivares 2000: J. C. Olivares, "Los dioses soberanos y los ríos en la religión indígena de la Hispania indoeuropea”, Gerión 18, 2000, 191-212.

Orduña 2014: E. Orduña, "Vocalismo átono en ibérico y romance", Liburna 7, 2014, 69-78.

Padrós 2002: P. Padrós, "El protagonisme de la moneda ibèrica a les ciutats romanes tardorepublicanes: Baetulo i la seca de Baitolo", en: VI Curs d'Història Monetària d'Hispània. Funció i producció de les seques indígenes, Barcelona 2002, 105-123.

Padrós 2005: P. Padrós, "Algunos ejemplos de la relación existente entre cecas ibéricas y fundaciones tardorepublicanas en el noreste de la Hispania Citerior", en: Actas XIII Congreso Internacional de Numismática, Madrid 2005, 523-530.

Padrós 2012: P. Padrós, "Las cecas layetanas. La ceca de Baitolo", en: A. G. Sinner (ed.), La moneda de los íberos. Ilturo y los talleres layetanos, Barcelona 2012, 44-54.

Purpura 2003: G. Purpura, Le Ancore. Archaeogate il portale italiano di archeologia, 2003. Accesible en: http://www1.unipa.it/dipstdir/portale/subacquea/ANCORE/index.html (consultado el 25 de julio del 2019).

Quero 2009: M. Quero, “Construcción de una réplica de ancla romana con cepo, zuncho y arganeo de plomo", Almoraima 39, 2009, 425-442.

Ripoll 1962: E. Ripoll, "Notas de arqueología de Cataluña y Baleares: Blanes”, Ampurias 24, 1962, 311-313. 
Ripollès y Sinner 2019: P. P. Ripollès y A. G. Sinner, "Coin evidence for ancient Hispanic languages”, en: A. G. Sinner y J. Velaza (eds.), Palaeohispanic languages and epigraphies, Oxford 2019, 365-395.

Rodríguez 2002: J. Rodríguez Ramos, “Problemas y cuestiones metodológicas en la identificación de los compuestos de tipo onomástico de la lengua ibera", Arse 36, 2002, 15-50.

Rodríguez 2005: J. Rodríguez Ramos, "Introducció a l'estudi de les inscripcions ibèriques", Revista de la Fundació Privada Catalana per l'Arqueologia ibèrica 1, 2005, 13-144.

Rodríguez 2014: J. Rodríguez Ramos, "Nuevo índice crítico de formantes de compuestos de tipo onomástico íberos", ArqueoWeb 15, 2014, 81-238.

Sabaté 2017: V. Sabaté, "Para un análisis de los compuestos onomásticos en plomos ibéricos: algunos ejemplos de su problemática”, en: Temas y tendencias actuales de la investigación. Actas de las II jornadas doctorales en ciencias de la antigüedad, Zaragoza 2017, 159-176.

Silgo 1994: L. Silgo, Léxico Ibérico, Valencia 1994.

Sinner y Ferrer 2016: A. G. Sinner y J.: Ferrer i Jané, "Del oppidum de Burriac a las termas de Ca l'Arnau. Una aproximación a la lengua y a la identidad de los habitantes de Ilduro (Cabrera de Mar, Barcelona)", AEspA 89, 2016, 199-223.

Sinner y Ferrer 2018: A. G. Sinner y J. Ferrer i Jané, “Novedades epigráficas de Ilduro (Cabrera de Mar, Barcelona)", PalHisp 18, 2018, 203-216.

Sinner y Ferrer e.p.: A. G. Sinner y J. Ferrer i Jané, “Baitolo, a native shipowner's vessel and the participation of the northern Iberians in the Laietanian wine trade during the late republican period", $J R A$, en prensa.

Torra 2009: F. Torra, "Les inscripcions ibèriques a la ciutat de Baetulo", Carrer dels arbres 20, 2009, 7-24.

Untermann 1990: J. Untermann, Monumenta Linguarum Hispanicarum Band III. Die iberischen Inschriften aus Spanien, Wiesbaden 1990.

Untermann 1998: J. Untermann, “La onomástica ibérica”, Iberia 1, 1998, 73-85.

Velaza 2011: J. Velaza, "El elemento -o en la formación de topónimos del área lingüística

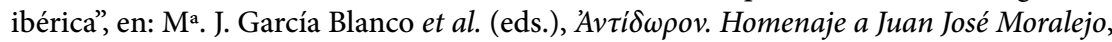
Santiago de Compostela 2011, 567-572.

Velaza 2015: J. Velaza, "Salaeco: un teónimo ibérico”, ZPE 194, 2015, 290-291.

Vidal 2016: J. C. Vidal, "Interpretació ibèrica de dos teònims preromans del nord-est peninsular", RAP 26, 2016, 195-204.

Vivar et al. 2013: G. Vivar, C. de Juan y R. Geli, "Cap del Vol. Un producto, un barco y un comercio del conventus Tarraconensis en época de Augusto", en: X. Nieto, A. Ramírez y P. Recio (eds.), I Congreso de Arqueología Náutica y Subacuática Española, Cartagena 2013, 97-108.

Zamora 1995: D. Zamora, Les ceràmiques de vernís negre del poblat ibèric del Turó d'en Boscà; aproximació a la interpretació històrico-arqueològica del poblat, Igualada 1995. 